\title{
Patterns of HRCT Findings and Its Clinical Correlation in Covid-19 Patients of a Tertiary Care Hospital in Assam
}

\author{
Nayan Mani Sarmaํ, Deep Kumar Roy², Rupak Bhuyan³ ${ }^{3}$ Mohsina Islam Bora ${ }^{4}$ \\ 1, 2,3,4 Department of Radiology, Jorhat Medical College and Hospital, Jorhat, Assam, India.
}

\section{ABSTRACT}

\section{BACKGROUND}

Covid-19, which is usually transmitted from droplet exposure, proved out to be a novel disease of human race. It is still unclear whether the new coronavirus can transmit in any other ways, but it can be detected in nasal swabs, sputum, respiratory secretions, blood, stool, and other samples. Early detection and prompt intervention play a vital role in reducing the number and severity of cases.

\section{METHODS}

Our study sample consisted of 100 patients from the Department of Radiodiagnosis, Jorhat Medical College \& Hospital (JMCH) diagnosed as Covid-19 from April 2020 to March 2021. Reviewing of chest CT images with clinical data was done and analysis of its relationship was reviewed. All the cases underwent thin-slice HRCT for at least a single time.

\section{RESULTS}

The most common CT findings observed were ground glass opacity (91\%), consolidation (63\%), and inter \& intra lobular septal thickening (59\%). We encountered multiple lesions, with an average of $13 \pm 5$ pulmonary segments infected.

\section{CONCLUSIONS}

Computed tomography thus, has a pivotal role in the prognostication of Covid-19 pneumonia which is an emergency global health epidemic. It helps us to detect and analyse the common chest manifestation of Covid-19 vis; consolidation, ground glassing, septal thickening, subpleural bands and occasional pleural effusions.
Corresponding Author: Dr. Mohsina Islam Bora, Department of Radiology, Jorhat Medical College and Hospital, Jorhat, Assam, India.

E-mail:mohsinabora@gmail.com

DOI: $10.14260 /$ jemds $/ 2022 / 2$

How to Cite This Article:

Sarma NM, Roy DK, Bhuyan $R$, et al. Patterns of HRCT findings and its clinical correlation in covid-19 patients of a tertiary care hospital in Assam. J Evolution Med Dent Sci 2021;11(01):7-12, DOI: $10.14260 / \mathrm{jemds} / 2022 / 2$

Submission 07-10-2021,

Peer Review 20-10-2021,

Acceptance 28-12-2021,

Published 05-01-2022.

Copyright (c) 2022 Nayan Mani Sarma et al. This is an open access article distributed under Creative Commons Attribution License [Attribution 4.0 International (CC BY 4.0)]

\section{KEY WORDS}

Covid-19, Pneumonia, Chest CT Scan, Ground Glassing, Interlobular Septal Thickening 


\section{BACKGROUND}

Covid-19, which is usually transmitted from droplet exposure, proved out to be a novel disease of human race. It is still unclear whether the new coronavirus can transmit in any other ways, but it can be detected in nasal swabs, sputum, respiratory secretions, blood, stool, and other samples. Early detection and prompt intervention play a vital role in reducing the number and severity of cases. Fever and common cold were the most commonly encountered clinical symptoms. The incidence of sputum in cough, chest discomfort, muscle pain, abdominal discomfort or loose stool, pharyngitis, light headedness and respiratory discomfort were less commonly seen. Furthermore, few of the cases show severe symptoms with complications like severe acute respiratory syndrome, renal failure, and even mortality. Some severe cases presented with dyspnoea and/or hypoxemia after 1 week of the onset of the disease, and develop rapidly into acute respiratory distress syndrome, septic shock, metabolic acidosis, and coagulation dysfunction, which are hard to correct. Currently there is only limited data available regarding the typical chest CT imaging findings in Covid-19. This is similar to other types of coronavirus infections such as the SARS and Middle East respiratory syndrome (MERS).1,2,3 Thus, it is concluded that they can provisionally be classified into the similar pattern of infectivity and the target cells of SARS-CoV-2 which is located in the lower respiratory tract. When comparing to other similar pneumonia it is found that Covid shows clinically milder symptoms with severe computed tomography findings. Vast majority of the patients presents with milder clinical symptoms like, minimal rise of temperature however, they had serious CT lung manifestations. In late December 2019, a pneumonia of unknown cause occurred as an outbreak in Wuhan city, Hubei province, China, with clinical symptoms similar to viral pneumonia including fever, cough, and dyspnoea. A previously unknown novel virus named as severe acute respiratory syndrome coronavirus 2 (SARSCoV-2) with a characteristic crown morphology at electron microscopy scanning was identified as the source of this disease. 4 Subsequently, the viral infection (pneumonia) caused by SARSCoV-2 was official termed as coronavirus disease 2019 (Covid-19) on 11 February $2020 . .^{5}$ As of March 13, 2020, a total of 81,003 patients with confirmed Covid-19, including 3181 deaths, have been reported in China. ${ }^{6}$ From current data of the cases, most patients with general and common symptoms have a relatively good prognosis while cases of death are more frequently seen in severe and critical patients, which could progress to severe pneumonia, ARDS and multiple organ failures leading to higher mortality. ${ }^{7}$ As a promising method recommended by the diagnosis and treatment program (7th trial edition) of National Health Commission of the People's Republic of China, ${ }^{8}$ high resolution computed tomography (HRCT) plays an essential role in the diagnosis and monitoring prognosis in Covid-19 patients. The main CT appearances of Covid-19 include ground glass opacities (GGOs) and patchy consolidations, which have respectively been regarded as a marker of early and progression stage of disease. ${ }^{9}$ Based on the above mentioned image abnormalities, HRCT could provide additional information to improve the planning of treatment, and evaluate the variations of image. To the best of our knowledge, few literatures focus on the change of CT findings among various clinical types during the course of medical treatment.

Therefore, the purpose of this study was to evaluate the discrepancy of series of CT manifestations in Covid-19 among different clinical types within the short term follow-up periods. Aiming to help clinicians monitor and predict outcome, to make more accurate and effective clinical decisions.

\section{METHODS}

This is a cross sectional study consisting of 100 patients from the Department of Radiodiagnosis, JMCH diagnosed as Covid19 from April 2020 to March 2021.

The inclusion criteria were (1) Epidemiological historyeither travel from outside Assam / residence history in Jorhat or exposure history to febrile patients suffering from respiratory symptoms within 14 days before the onset of illness and (2) laboratory diagnosis (RT-PCR revealed positive detection of SARS-CoV-2 in throat swabs or lower respiratory tract).

The criteria for exclusion were another microbiologically positive non Covid -19 pulmonary pneumonia. Young children and women with gravid uterus were not included in this study.

- $\quad$ All the cases had undergone the CT examinations using the DUAL SLICE CT scanner (Siemens Somatom Sensation; Siemens, Erlangen, Germany) using the following parameters: $120 \mathrm{kVp}, 150 \mathrm{~mA}, 1.5 \mathrm{~mm}$ collimation, 1.35:1 pitch, slice thickness of $3.0 \mathrm{~mm}$, with high spatial resolution algorithm. All the patients were positioned in supine position during proper breathholding at the peak of inspiration.

- The CT imaging features were totally analysed, and the following parameters were computed: ground glass opacity, consolidation, interlobular septal thickening, bronchial wall thickening, subpleural line, lymph node enlargement, pleural effusion and pericardial effusion in accordance with the standard morphologic descriptors based on the Fleischner Society Nomenclature Committee recommendations and similar studies. ${ }^{10,11}$

\section{Statistical Analysis}

All statistical analyses in the study were analysed using Statistical Package for Social Sciences software version 23.0 (SPSS Inc, Chicago, IL).

\section{RESULTS}

The study included 100 patients with Covid-19 positive status, of whom males were $52 \%$ and females were $48 \%$. The age group of patients was 16 to 80 years with an average age of $45 \pm 10$ years. 33 patients were smokers. 76 patients had rise in temperature, 18 patients had associated morbidities, 5 patients had history of increased blood pressure, 5 patients had history of hyperglycaemia, 4 patients had emphysema, 3 patients had high risk behaviour with suppression of 
immunity (intravenous venous drug users), and 1 patient had cardiac ailments (coronary artery disease).

No patients gave a history of obstructive lung disease or chronic allergic lung manifestations. In view of the signs and symptoms, 72 of the 100 patients had sore throat, 56 patients had rise in temperature, 14 patients had sputum on cough, 6 patients had chest discomfort, 16 patients had muscle pain, 9 patients had abdominal upset or loose stool, 11 patients had pharyngeal irritation, 10 patients had mild lightheaded ness. Regarding the laboratory, total lymphocyte count was $1.15 \mathrm{x}$ 109/L (IQR, 0.76-1.40 x 109/L).

\section{Chest CT Findings}

All the cases had undergone computed tomography of chest 8 \pm 3 days after the initiation of the disease. 95 cases presented with pneumonic chest CT abnormalities. Ground glass opacities were found to be the most frequent computed tomography findings in 91 cases, 63 cases presented with consolidation, 59 cases presented with interstitial thickening, (Fig. 1). Maximum of the lung abnormality found to be multiple, with an average involvement of $13 \pm 6$ lung segments. 53 patients showed subpleural distribution, 9 patients showed diffuse involvement, 4 patients showed peri bronchial distribution, 30 patients showed mixed distribution.

The most frequently involved lung segments were the right lower lobar superior segment $(86 / 100$,) the right lower lobar posterior basal segment (85/100), right lower lobar lateral basal segment (80/100), left lower lobar superior segment $(76 / 100)$, and the left lower lobar posterior basal segment (81/100). Besides these 3 radiological findings, 11 patients had thickening of bronchial wall, 20 patients had curvilinear lines in subpleural plane, 6 cases had occasional fluid in the pleural cavity, 4 cases had lymphadenopathy, and 5 cases had fluid in the pericardial cavity. The characteristic signs were "crazy paving sign" (29/100) and "spider web sign" (25/100) (Figs. 2, 3).

The "crazy paving pattern" is defined by the interstitial reticular thickening with the patchy ground glassing, which are found in cases of severe acute respiratory syndrome (SARS) literatures.

The "spider web sign" is the opacity encountered for the very first time. This sign is defined as a triangular or wedgeshaped ground glass opacity in the subpleural plane associated with interstitial septal thickening resembling a net. The pleura in its vicinity were distorted and pulled to form a spider web-like shape.

\begin{tabular}{|cc|}
\hline Age & 44 (Average) \\
Sex & Male- 52, female- 48 \\
Smokers & 30 \\
Comorbidities & $\begin{array}{c}21 \text { (HTN- 7, diabetes- 8, COPD-4, } \\
\text { immunosuppresion-3, cardiac ailments- 1, } \\
\text { bronchial asthma-2) }\end{array}$ \\
$\begin{array}{c}\text { Fever, rise of temperature, cough, } \\
\text { expectoration, chest pain }\end{array}$ & $\begin{array}{c}72 \text { (fever), 37.80-degree Celsius } \\
\text { (average temperature), 70 (cough), } 21 \\
\text { (expectoration), 8 (chest pain). }\end{array}$ \\
\hline Table 1. Characteristics and Clinical Manifestations in 100 Patients \\
with Covid-19
\end{tabular}
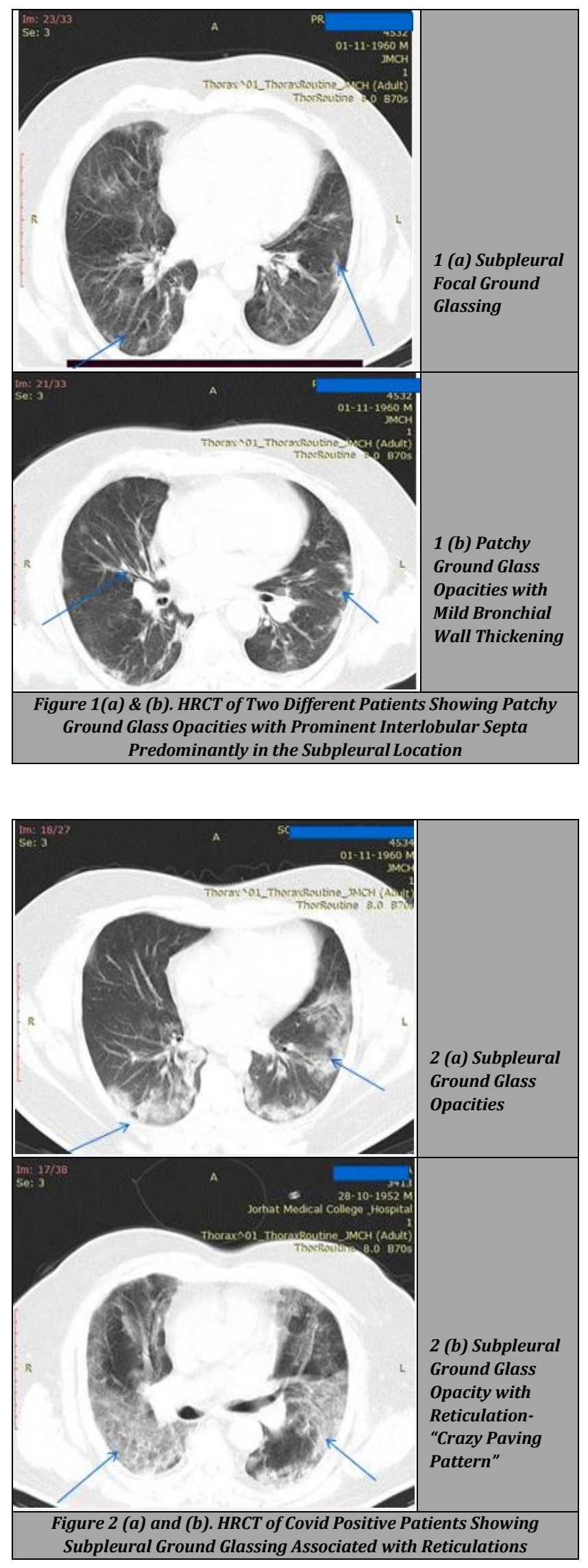

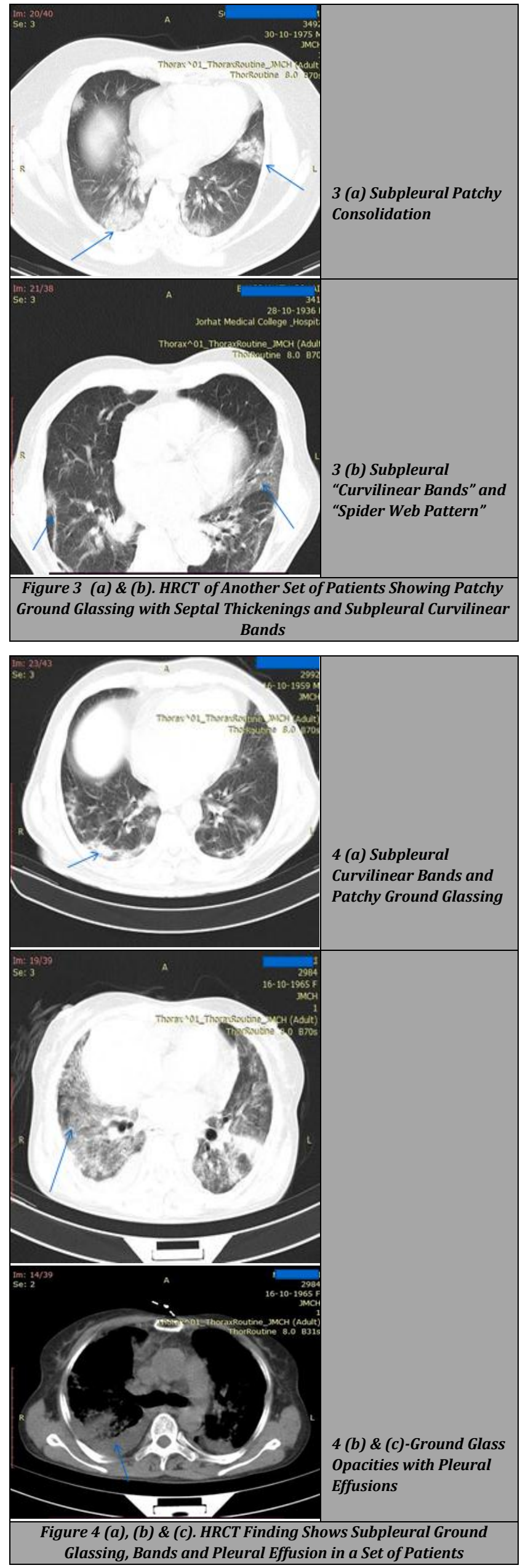

\begin{tabular}{|cc|}
\hline WBC count $(5.4 \times 1000 / \mathrm{dl})$ - increased / decreased & 10 (increased), 7 (decreased) \\
Neutrophil count $(3.74 \times 1000 / \mathrm{dl})$ - increased / & 20 (increased), 6 (decreased). \\
decreased & 43 (decreased) \\
Lymphocyte count $(1.15 \times 1000 / \mathrm{dl})$ & 46 (increased) \\
CRP & 123 \\
Systolic pressure, $\mathrm{mmHg}$ & 80 \\
Diastolic pressure, $\mathrm{mm} \mathrm{Hg}$ & $88 \& 21$ \\
Heart rate \& respiratory rate & \\
\hline Table 2. Laboratory and Physical Examination Results of 100 Patients \\
with Covid-19
\end{tabular}

\begin{tabular}{|cc|}
\hline CT Features & \\
Ground glassing & 86 \\
Consolidation & 58 \\
Interstitial thickening & 54 \\
Crazy pavement pattern & 31 \\
Spider web sign & 21 \\
Subpleural line & 20 \\
Peri bronchial cuffing & 9 \\
Pleural effusion & 6 \\
Lung segment involved & 86 \\
Right lower lobar superior segment & 80 \\
Right lower lobar lateral basal segment & 85 \\
Right lower lobar posterior basal segment & 76 \\
Left lower lobar superior segment & 81 \\
\hline Left lower lobar posterior basal segment & \\
\hline Table 3. Chest CT Findings of 100 Patients with Covid-19 \\
\hline
\end{tabular}

\section{DISCUSSION}

In our study, maximum patients we encountered were adults, without any significant difference between the numbers of male or female patients. Most commonly encountered symptoms were common cold and rise of temperature. The sputum on cough, chest discomfort and muscle pain were less frequently encountered. Maximum cases showed severe lung computed tomography manifestations corresponding to milder clinical signs and symptoms. Various segments showed multiple morphological changes in both the lungs, different from the bacterial pneumonia. Radiologically computed tomography features like ground glass opacities were the most frequently encountered abnormality, associated with interstitial septal thickening and consolidations.

Few of the recently published studies shows concomitant findings. ${ }^{12,13}$ In few cases we can see the subpleural lines and peri bronchial cuffing, with rarely visualized fluid in the pleural cavity, fluid in the pericardial cavity, without any significant lymph node enlargement. Ground glass opacity with interstitial reticular thickening, named as "crazy paving pattern" are encountered in large number of cases. Few of the ground glass opacities appear triangular or wedge shaped predominantly in the subpleural plane associated with the interstitial thickening giving rise to "Spider web sign" configuration. ${ }^{14}$ This is a very specific sign for Covid-19, which is not explained for any other lung diseases in the literature. At present, it is not clear whether it has clinical relevance in evaluating the prognosis of the cases. Recently, Pan et al. have analysed the time course of lung changes in 21 patients with mild symptoms with confirmed Covid-19. They found that the initial lung manifestation on CT was subpleural GGO, which changed to consolidation 2 weeks after the onset of the disease, and then the lesions gradually subsided, leaving a wide spectrum of GGO and subpleural parenchymal bands. ${ }^{15}$ In another article, the authors found that after 7 days of treatment for the mild symptomatic patients, there was a significant resorption in GGO on chest CT. The Covid-19 
needs to be differentiated from other diseases, such as SARS and MERS. They all showed multiple ground glass shadows and solid lesions in both lungs, mainly distributed under the pleura, which were difficult to distinguish. ${ }^{16,17,18}$ It is very vital to differentiate Covid -19 from other pneumonia like, such as SARS and MERS. They all showed multiple ground glass shadows and solid lesions in both lungs, mainly distributed under the pleura, which were difficult to distinguish. It is found that SARS and MERS presented with very severe lung destruction with protracted course when comparing to the Covid-19 pneumonia. Similarly, Covid-19 also need to be differentiated from other various viral pneumonia such as influenza virus, parainfluenza virus, adenovirus, respiratory syncytial virus, rhinovirus, human metapneumovirus, and mycoplasma pneumonia. At this current stage as per our knowledge, all Covid cases have not undergone histo-pathological examination around the world, so it not possible to get the proper histopathological findings of Covid-19 pneumonia. Moreover, as per the morphological pattern of the CT chest findings, histo-pathological findings could be analysed virtually without proper direct evidences. The ground glass opacity lesions might be due to the accumulation of exudate in the alveoli. The pulmonary consolidations found in HRCT thorax are defined as an opacity where the alveoli are completely filled with exudates.

\section{CONCLUSIONS}

We can conclude that computed tomography plays a pivotal role in the investigation of Covid 19 pneumonia which is an emergency global health epidemic. It helps us to detect and analyse the most common chest manifestation of Covid-19 vis; ground glass densities, consolidations and interstitial septal thickening which are predominantly in the subpleural location. There is a close correlation between the severity of pulmonary infection on CT scans and the clinical manifestations with laboratory investigations. Thus, computed tomography played a pivotal role in the diagnosis and prognostication of this emerging global health epidemic.

\footnotetext{
Limitations

Firstly, our study was a retrospective study as we considered a small number of patients with microbiological proven Covid-19 pneumonia. Next, none of the cases had undergone a proper HPE diagnosis either FNA /biopsy of lung. Also, in our study we failed to follow up the cases for further lung changes as our study was a cross-sectional study. In the near future, we shall try to evaluate the chest CT findings of different stages of Covid-19 by choosing larger and also diversified samples. Moreover, as large Covid-19 infection passes through various phases from the acute to subacute stages progressing to a completely cured infection or to a more chronic complicated phase in few patients in upcoming several days and months which will be analysed in many future studies. Those studies will innumerate any long-term chronic complications that are currently absent in our study (e.g., fluid in pleural cavity, infected pleural collections, enlarged lymph nodes and cavity in lung). The preliminary and long term follow-up of HRCT chest findings of Covid-19
}

changes depending upon the clinical manifestation. The severe diseases in chest CT shows clinically less severe manifestations and improvement after initial treatment, while few cases aggravated on follow up. Thus, HRCT thorax help us to diagnose and prognosticate the severity of Covid19 pneumonia, and guide us to follow up the cases with various clinical manifestations.

Data sharing statement provided by the authors is available with the full text of this article at jemds.com.

Financial or other competing interests: None.

Disclosure forms provided by the authors are available with the full text of this article at jemds.com.

\section{REFERENCES}

[1] Lee N, Hui D, Wu A, et al. A major outbreak of severe acute respiratory syndrome in Hong Kong. N Engl J Med 2003;348(20):1986-94.

[2] Assiri A, Al-Tawfiq JA, Al-Rabeeah AA, et al. Epidemiological, demographic, and clinical characteristics of 47 cases of Middle East respiratory syndrome coronavirus disease from Saudi Arabia: a descriptive study. Lancet Infect Dis 2013;13(9):752-61.

[3] Muller NL, Ooi GC, Khong PL, et al. High-resolution CT findings of severe acute respiratory syndrome at presentation and after admission. Am J Roentgenol 2004;182(1):39-44.

[4] WHO. Naming the coronavirus disease (COVID-2019) and the virus that causes it. Geneva: World Health Organization 2020.

[5] WHO. WHO Director-General's remarks at the media briefifing on 2019- CoV on 11 February 2020. Geneva: World Health Organization 2020

[6] Health Emergency Offifice of National Health Commission of the PRC. Update on the epidemic of novel coronavirus (2019-nCoV) infected pneumonia as at 24:00 on 13 March, 2020.

[7] Li Q, Guan X, Wu P, et al. Early transmission dynamics in Wuhan, China, of novel coronavirus-infected pneumonia. N Engl J Med 2020;382(13):1199-207.

[8] National Health Commission of the People's Republic of China. Diagnosis and treatment scheme for coronavirus disease 2019 (Trial Version 7). 3.5;2020.

[9] Chinese Society of Radiology. Radiological diagnosis of new coronavirus infected pneumonitis: expert recommendation from the Chinese Society of Radiology (First edition). Chin J Radiol 2020;54:E001.

[10] Hansell DM, Bankier AA, MacMahon H, et al. Fleischner society: glossary of terms for thoracic imaging. Radiology 2008;246(3):697-722.

[11] Schoen K, Horvat N, Guerreiro NFC, et al. Spectrum of clinical and radiographic findings in patients with diagnosis of $\mathrm{H} 1 \mathrm{~N} 1$ and correlation with clinical severity. BMC Infect Dis 2019;19(1):964.

[12] Song FX, Shi NN, Zhang ZY, et al. Emerging 2019 novel coronavirus (2019-nCoV) Pneumonia. Radiology 2020;295(1):210-7.

[13] Kanne JP. Chest CT findings in 2019 novel coronavirus (2019-nCoV) infections from Wuhan, China: key points for the radiologist. Radiology 2020;295(1):16-7. 
[14] Wu J, Wu X, Zeng W, et al. Chest CT findings in patients with coronavirus disease 2019 and its relationship with clinical features. Invest Radiol 2020;55(5):257-61.

[15] Pan F, Ye TH, Sun P, et al. Time Course of Lung Changes at Chest CT during Recovery from Coronavirus Disease 2019 (Covid-19). Radiology.2020;295(3):715-721.

[16] Das KM, Lee EY, Enani MA, et al. CT correlation with outcomes in 15 patients with acute Middle East respiratory syndrome coronavirus. AJR Am J Roentgenol 2015;204(4):736-42.
[17] Ajlan AM, Ahyad RA, Jamjoom LG, et al. Middle East respiratory syndrome coronavirus (MERS-CoV) infection: chest CT findings. AJR Am J Roentgenol 2014;203(4):782-7.

[18] Hui DS, Memish ZA, Zumla A. Severe acute respiratory syndrome vs. the Middle East respiratory syndrome. Curr Opin Pulm Med 2014;20(3):233-41. 